\title{
Percepção Material do Porongo (Lagenaria siceraria) em relação à Madeira e Derivados
}

Bottle Gourd (Lagenaria siceraria) Material Perception in relation to Woods and Derivatives

NEJELISKI, Danieli; Mestra em Design; Universidade Federal do Rio Grande do Sul; Brasil danielinejeliski@gmail.com

DUARTE, Lauren da Cunha; Doutora; Universidade Federal do Rio Grande do Sul; Brasil

lauren.duarte@ufrgs.br

PICCOLI, Mariana; Mestra em Design; Universidade Federal do Rio Grande do Sul; Brasil

Marianap.piccoli@gmail.com

\section{Resumo}

O porongueiro (Lagenaria siceraria) é uma planta de hábito tropical disseminada em todos os continentes, com ciclo produtivo anual e alta produtividade. Após o processo de secagem, os frutos são utilizados para a produção de recipientes para alimentos e outros artefatos. Compreender como os usuários percebem o material é fundamental para ampliar as possibilidades de aplicação no design de novos produtos. O objetivo do estudo é identificar a percepção material do porongo com relação à madeira e derivados. A análise foi realizada a partir de nove amostras: duas de madeira, seis de derivados e uma de porongo. Os materiais foram avaliados através de um questionário com atributos sensoriais e outro com atributos semânticos. O porongo obteve pontuações altas nos atributos sensoriais de leve, liso e macio, bem como nos atributos semânticos de natural, sustentável, delicado e agradável. O estudo demonstrou que as principais características do material são identificadas pelo usuário.

Palavras Chave: Lagenaria siceraria; percepção sensorial; percepção semântica.

\begin{abstract}
The bottle gourd (Lagenaria siceraria) is a plant with tropical habit disseminated in all continents, with annual productive cycle and high productivity. After the drying process, the fruits are used for the production of food containers and other artifacts. Understanding how users perceive the material is critical to expanding application possibilities in new product design. The objective of the study is to identify the material perception of bottle gourd in relation to wood and derivatives. The analysis was carried out from nine samples: two of wood, six of derivatives and one of bottle gourd. The materials were evaluated through a questionnaire with sensorial attributes and another with semantic attributes. The bottle gourd obtained high scores on the sensory attributes of light, smooth and soft, as well as on the semantic attributes of natural, sustainable, delicate and pleasant. The study demonstrated that the main characteristics of the material are identified by the user.
\end{abstract}

Keywords: first keyword; second keyword; third and last keyword. 


\section{Introdução}

O porongo, de nome científico Lagenaria siceraria, é o fruto de uma planta da família das cucurbitáceas, relacionada com a origem da agricultura e das civilizações, estando entre as primeiras espécies a serem domesticadas (BISOGNIN, 2002). Exemplares que datam de 11.000 AC foram encontrados na Ásia Oriental. No continente americano, eram utilizados desde pelo menos 10.000 AC (KISTLER et al., 2014). Planta de hábito tropical, disseminou-se por todos os continentes. Seu ciclo produtivo é anual e a produtividade média pode ultrapassar os 10 mil frutos por hectare (BISOGNIN et al.,1992).

A colheita é realizada após a senescência natural da planta, em que os frutos são colhidos e amontoados à sombra para secar lentamente. $O$ processo de secagem pode demorar mais de seis meses para permitir a cura do material. O verde da epiderme exterior irá desaparecer e o fruto perderá $90 \%$ do seu peso com a evaporação da água presente, tornando-se oco (BURTENSHAW, 2003). A parte interna da casca adquire características semelhantes à da madeira e a casca externa, extremamente fina e lisa, torna-se impermeável e quando polida adquire brilho acentuado.

Em virtude de suas características formais é utilizado como matéria-prima para a produção artesanal de recipientes para alimentos e bebidas, especialmente na África. Suas excelentes propriedades acústicas são exploradas há milhares de anos na confecção de instrumentos musicais (BURTENSHAW, 2003). O cultivo do porongo é uma importante atividade agrícola no estado do Rio Grande do Sul, onde o fruto é utilizado na fabricação de cuias para chimarrão, bebida típica da região (BISOGNIN; SILVA, 2004). A utilização do fruto como matéria-prima para a confecção de produtos é essencialmente artesanal. Entretanto, estudos relacionados à impermeabilização do material para aumento da durabilidade e posterior aplicação em escala industrial foram realizados, como o uso do material na produção de biojoias (LAGO, 2013) e a impermeabilização da parte interna do material com resina poliuretana de óleo de mamona para a produção de recipientes para alimentos e bebidas (NEJELISKI, 2015).

A utilização de materiais naturais pela indústria vem aumentando gradativamente em virtude das reais possibilidades de renovação das reservas, pela biocompatibilidade relacionada à facilidade de absorção pela natureza quando descartados e, nas aplicações onde há o contato humano, os materiais naturais são considerados mais confortáveis (LIMA, 2006). O porongo é uma matéria-prima com ciclo produtivo anual, altamente renovável, vinculado à agricultura familiar e com elevado potencial de aplicação em novos produtos. Entretanto, a introdução de novos materiais no mercado está relacionada como a capacidade de prever para quais aplicações o mesmo será adequado. Para isso, é fundamental compreender a percepção que o usuário final tem do material antes de aplicá-lo em novos produtos.

Nesse contexto, Ashby e Johnson (2011) afirmam que, inicialmente, um material não tem atributos percebidos como indiscutíveis. Entretanto, com o passar do tempo, vai adquirindo significados quando aplicado em produtos e, assim, pode incorporar uma personalidade. A madeira, por exemplo, é um material visual e tátil, tem textura, desenhos, é percebida como mais quente, desperta associações com a natureza, com o trabalho manual. Portanto, há um caráter oculto em um material, antes mesmo de ele adotar uma forma reconhecível, "um tipo de personalidade embutida, tímida que seja, nem sempre visível, fácil de ocultar ou disfarçar, mas que, quando adequadamente manipulada, pode contribuir para o bom design" (ASHBY; JOHNSON, p. 82, 2011). 
Para Hartzén e Lindberg (2016) a expressão de um material não é fixa, mas dinâmica. Uma série de fatores influenciam a maneira como os usuários percebem um material. Gênero, contexto social, cultura, grau de instrução e mesmo o fato de o material estar ou não associado a um produto específico. Ainda, no processo de reconhecimento, os sentidos da visão e do tato se complementam e dão origem a diferentes tipos de associações quando experimentamos materiais. Apesar da expansão da área de Engenharia de Materiais e do investimento em pesquisa no setor, de acordo com Karana e Nijkamp (2014) ainda existe uma lacuna na compreensão de como os novos materiais são avaliados pelos usuários. Estudos de percepção dos novos materiais em relação a outros já consolidados se constituem em uma alternativa para amenizar esta lacuna. Assim, este estudo tem como objetivo analisar a percepção material do porongo com relação à madeira e derivados, a fim de identificar uma possível demanda de aplicação do mesmo em novos produtos.

\section{Materiais e Métodos}

Para analisar a percepção material do porongo, optou-se por compará-lo com a madeira e alguns dos seus principais derivados, por serem materiais com os quais os usuários têm mais contato no cotidiano. Foram confeccionadas amostras de duas espécies de madeira, Cedro e Pinus. Com relação aos materiais derivados, foram utilizados o compensado laminado revestido com lâminas de madeira, o aglomerado sem revestimento, o Medium Density Fiberboard - MDF, painel de fibras de madeira de média densidade, nas opções sem revestimento e revestido com melamina branca e com textura amadeirada. Ainda na categoria de derivados, foi utilizada uma amostra de madeira plástica, compósito desenvolvido a partir da reutilização de resíduos orgânicos aglutinados com resina. Por fim, foi utilizada uma amostra de porongo.

Ao todo, foram confeccionadas nove amostras com dimensões de $8 \mathrm{~cm} \times 4 \mathrm{~cm}$, para facilitar o manuseio e a análise por parte dos participantes. Os materiais não foram identificados, apenas as amostras enumeradas e dispostas em uma ordem fixa (figura 1), primeiro foram analisadas as duas amostras de madeira, depois os derivados e por fim o porongo, com o intuito de facilitar a comparação do mesmo com demais materiais.

Foram realizados dois estudos de percepção material, os quais concentraram-se em dois tipos de avaliações de usuários: propriedades sensoriais e significado afetivo usando uma abordagem semântica de produto, tendo como referência o estudo desenvolvido por Hartzén e Lindberg (2016). 
Figura 1 - Relação das amostras: Cedro, Pinus, compensado laminado, aglomerado sem revestimento, MDF sem revestimento, MDF revestido com melamina branca, MDF revestido com melanina amadeirada, madeira plástica e porongo

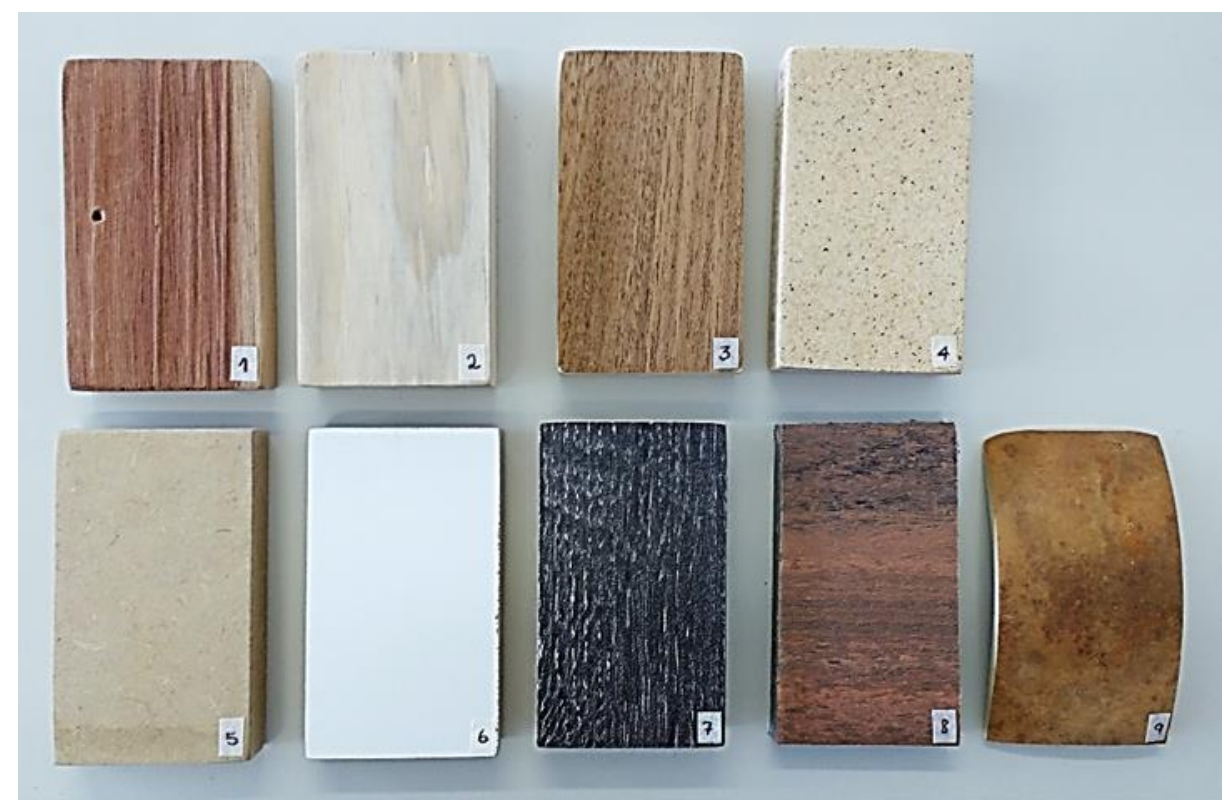

Fonte: os autores

Os atributos da escala sensorial foram definidos com base na proposta das autoras supracitadas, com algumas adaptações. A ordem em que os atributos aparecem foi alterada para que as propriedades relacionadas ao tato fossem apresentadas em primeiro lugar e as propriedades visuais na sequência, a fim de tornar mais fácil para os entrevistados compreender qual o domínio sensorial predominante na avaliação de cada propriedade. No questionário, os atributos aparecem organizados em pares de opostos, avaliados em uma escala de 1 a 5 pontos inteiros, onde quanto mais próximo dos extremos, mais intensa a característica. Assim, foram elencados oito pares de características sensoriais a serem analisados: duro/macio, áspero/liso, frio/quente, elástico/rígido, fraco/forte, pesado/leve, opaco/brilho e sem textura/com textura.

Já os atributos da escala semântica foram definidos com base na lista dos atributos percebidos nos produtos de Ashby e Johnson (p. 82, 2011). Da mesma maneira que na escala sensorial, na escala semântica os atributos foram organizados aos pares de características opostas, em uma escala de 1 a 5 pontos inteiros. Os termos artificial/natural foram acrescentados a fim de verificar se a origem do material afeta a forma como os entrevistados percebem os mesmos (Hartzén; Lindberg, 2016). O conjunto final de palavras inclui os seguintes pares, na ordem: artificial/natural, não sustentável/sustentável, descartável/duradouro, grosseiro/delicado, comum/exclusivo, discreto/extravagante, barato/caro, desagradável/agradável.

Os dados foram coletados a partir de dois questionários online, cada qual respondido por um grupo de 14 voluntários diferentes. A razão para consultar grupos distintos para a análise sensorial e a semântica foi a de evitar a transferência de informações entre os estudos. As análises foram conduzidas em uma sala com ambiente neutro. Os participantes foram orientados a sentar em uma mesa com um notebook para responder ao questionário e as amostras dispostas ao lado do mesmo (figura 2). Foram instruídos a avaliar uma amostra de cada vez, respeitando a ordem numérica, responder o questionário, para então passar para a próxima amostra. Eles estavam livres 
para tocar e olhar para os materiais como quisessem.

Figura 2: Procedimento do estudo: análise sequencial de cada amostra seguida do preenchimento do questionário online

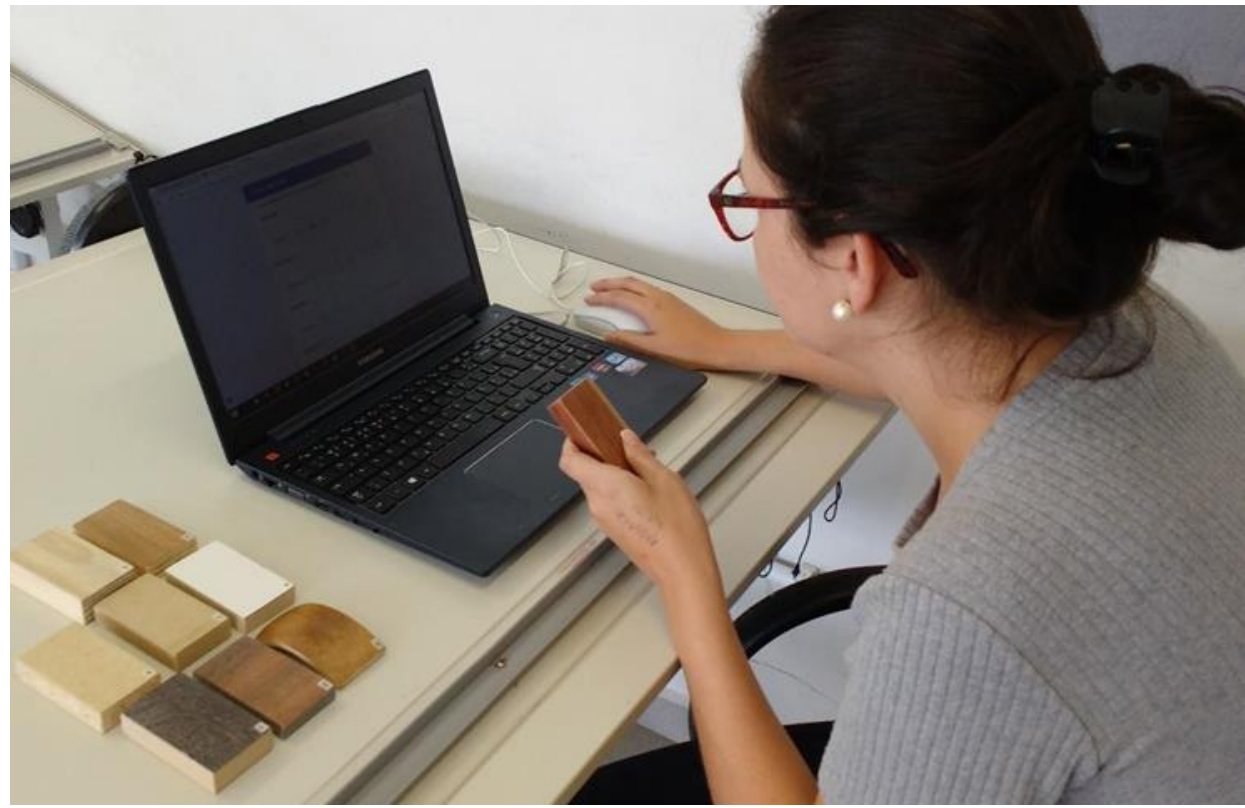

Fonte: os autores

O grupo de participantes do estudo foi composto por voluntários do Curso Técnico em Móveis Subsequente e do Bacharel em Arquitetura e Urbanismo, do Instituto Federal Farroupilha IFFar, Campus Santa Rosa. A média de idade dos respondentes foi de 25 anos. Participaram do estudo sensorial nove mulheres e cinco homens. Já no estudo semântico participaram onze mulheres e três homens.

\section{Resultados}

Para estimar o grau de concordância entre as respostas dos participantes em cada questionário, foi utilizada a média aritmética e a correlação de Pearson (tabela 1). De um modo geral, a consistência entre as respostas foi baixa nos dois estudos, na escala sensorial a média foi de $r=0,30$, e na escala semântica a média foi de $r=0,24$. A maior coerência entre as respostas da escala sensorial pode ser explicada pelo fato destes atributos serem mais palpáveis, já que são avaliados a partir do tato e da visão, enquanto que os atributos semânticos podem ser considerados mais subjetivos, dependem da interpretação individual de seu significado.

Analisando os dois estudos separadamente, na escala sensorial dois atributos apresentaram valores significativamente acima de zero, liso e leve, o que significa que os participantes foram bem consistentes uns com os outros nestes dois critérios. Em contrapartida, dois atributos apresentaram valores muito baixos, macio e rígido, o que demonstra que não houve consenso na interpretação e na análise das amostras frente a estes atributos. Já na escala semântica, os atributos com a correlação mais alta foram natural e agradável, indicando coerência nas respostas e relação entre os termos. O atributo extravagante obteve uma correlação próxima de zero, o que pode ser 
explicado por incoerência na interpretação do significado do termo, que pode parecer dúbio para muitas pessoas. Outro atributo inconsistente nas respostas foi delicado, característica que normalmente não está associada aos materiais em questão, o que pode ter contribuído para a baixa correlação.

Tabela 1: Atributos utilizados para os estudos sensorial e semântico e a respectiva correlação das respostas

\begin{tabular}{cccc}
\hline Descrição sensorial & Consistência das respostas & Descrição semântica & Consistência das respostas \\
& $(\mathbf{r})$ & Natural & $(\mathbf{r})$ \\
\hline Macio & 0,14 & Sustentável & 0,51 \\
\hline Liso & 0,58 & Duradouro & 0,08 \\
\hline Quente & 0,21 & Delicado & 0,36 \\
\hline Rígido & 0,11 & Exclusivo & 0,13 \\
\hline Forte & 0,33 & Extravagante & 0,19 \\
\hline Leve & 0,50 & Caro & 0,08 \\
\hline Brilho & 0,32 & Agradável & 0,19 \\
\hline Textura & 0,22 & & 0,43 \\
\hline
\end{tabular}

Fonte: dos autores

Para uma melhor visualização e apreciação dos resultados, os índices médios de cada estudo - sensorial e semântico - foram separados por grupos de materiais. Assim, foi compilado um gráfico para a média das duas amostras de madeira, um para a média do grupo dos derivados da madeira - compensado laminado, aglomerado e amostras de MDF. A média dos resultados da madeira plástica e do porongo foram considerados isoladamente, cada qual com um gráfico a parte. A partir da observação dos gráficos (figuras 3 e 4), pode-se perceber que os diferentes grupos de materiais tendem a ter diferentes perfis.

Com relação aos atributos sensoriais (figura 3), as amostras de madeira obtiveram pontuação alta no que se refere à rigidez e à leveza, condizente com o senso comum de que a madeira é um material resistente e leve. $O$ atributo brilho recebeu pontuação significativamente baixa. Já o grupo dos derivados obteve uma pontuação média muito similar à madeira em alguns aspectos. A pontuação média dos atributos macio, liso, rígido, forte e textura foi praticamente a mesma para os dois grupos de materiais, o que reforça o objetivo do desenvolvimento e da aplicação desta categoria de materiais, que é justamente configurar-se como uma alternativa de material similar à madeira para suprir a demanda do mercado.

A madeira plástica é um material relativamente novo no mercado brasileiro, e vem ganhando espaço como substituto da madeira em aplicações em produtos para áreas externas, pois tem maior durabilidade quando em contato com intempéries. Optou-se por apresentar as pontuações do material em um gráfico a parte pois o mesmo difere dos demais derivados da madeira utilizados no estudo com relação ao percentual de polímero utilizado na produção do compósito, que é consideravelmente maior do que nos demais. Por isso a madeira plástica tem mais características táteis e visuais da família dos polímeros do que dos materiais naturais. 0 material obteve pontuação significativamente alta no atributo textura, o que pode ser explicado pelo 
resquício do uso de resíduos orgânicos, como galhos e cascas de árvore, serragem e outros, que ficam visíveis. $O$ atributo rígido também obteve uma pontuação alta, entretanto, mais baixa do que nas madeiras e derivados.

Já a análise dos atributos sensoriais do porongo possui dois aspectos que se destacam dos demais em função da alta pontuação. Os atributos liso e leve se aproximaram da pontuação máxima, 4,8 e 4,9, respectivamente, em uma escala de 1 a 5 . Esse resultado é relevante pois demonstra que a leveza do material é percebida pelo usuário, sendo essa uma de suas principais propriedades. Em estudo realizado para a determinação das propriedades básicas do porongo, a densidade básica do mesmo foi estimada em $0,107 / \mathrm{cm}^{3}$, menor do que da madeira balsa, considerada a de menor densidade, e muito similar à densidade de algumas espumas poliméricas (NEJELISKI, 2015). A percepção do material como sendo muito liso deve-se ao aspecto homogêneo da sua casca. $\mathrm{O}$ atributo de maciez também recebeu pontuação alta, o que pode estar associado à textura da parte interna do material. Outro aspecto que cabe destacar é a pontuação do atributo quente, muito similar à madeira. Em contraponto, a pontuação do atributo forte foi consideravelmente baixa, o que pode ser explicado pelo fato de os participantes interpretarem leveza e resistência como contraditórios.

Figura 3: Pontuação média dos atributos sensoriais para cada grupo de materiais: madeiras, derivados, madeira plástica e porongo
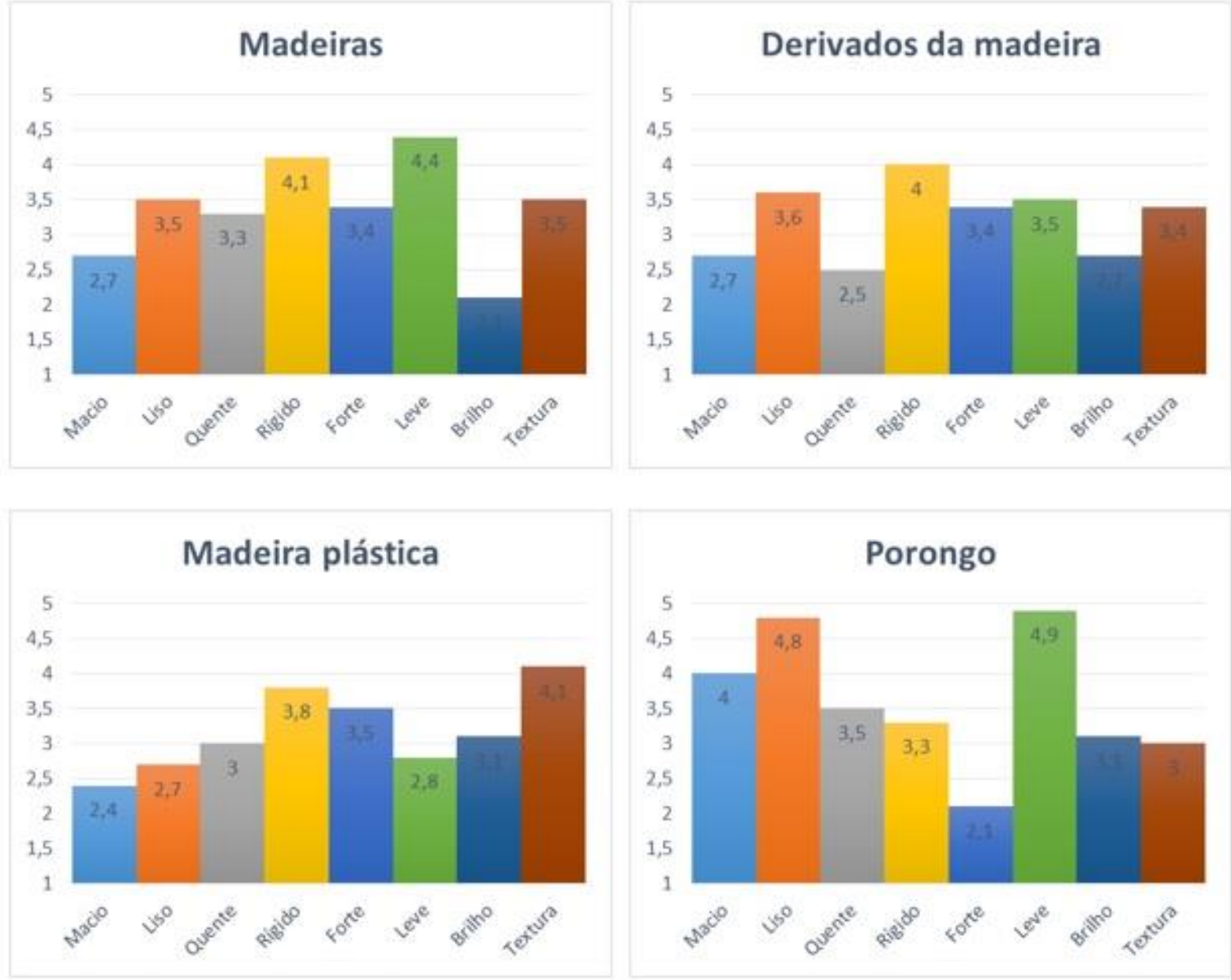

Fonte: dos autores 
Já com relação aos atributos semânticos (figura 4), a análise das amostras de madeira ficou dentro do que era esperado. Os atributos natural, sustentável, duradouro e agradável obtiveram uma pontuação alta, com média de 4,0 pontos, o que reforça a relação que o usuário faz automaticamente entre estes quatro aspectos. Se o material é natural, entende-se que é sustentável e duradouro, e essa associação de material ecologicamente correto faz com que seja percebido como esteticamente agradável. Em contraponto, os materiais derivados da madeira obtiveram pontuação relativamente mais baixa nestes quatro atributos - natural, sustentável, duradouro e agradável. Ainda, o atributo exclusivo obteve pontuação baixa, o que pode estar associado ao fato de produtos produzidos a partir de materiais derivados, especialmente o MDF, estarem mais presentes no cotidiano dos participantes do que a própria madeira, sendo assim estes materiais são percebidos como banais.

A madeira plástica é visivelmente percebida como um material artificial, sendo a pontuação no atributo natural muito perto do mínimo. Entretanto, foi identificada como um material sustentável, com uma pontuação consideravelmente alta neste atributo, o que pode ser justificado pelo fato de o uso de resíduos na produção ser perceptível na análise visual e tátil do material. Os atributos duradouro, exclusivo e agradável também obtiveram pontuação relativamente alta.

Figura 4: Pontuação média dos atributos semânticos para cada grupo de materiais: madeiras, derivados, madeira plástica e porongo

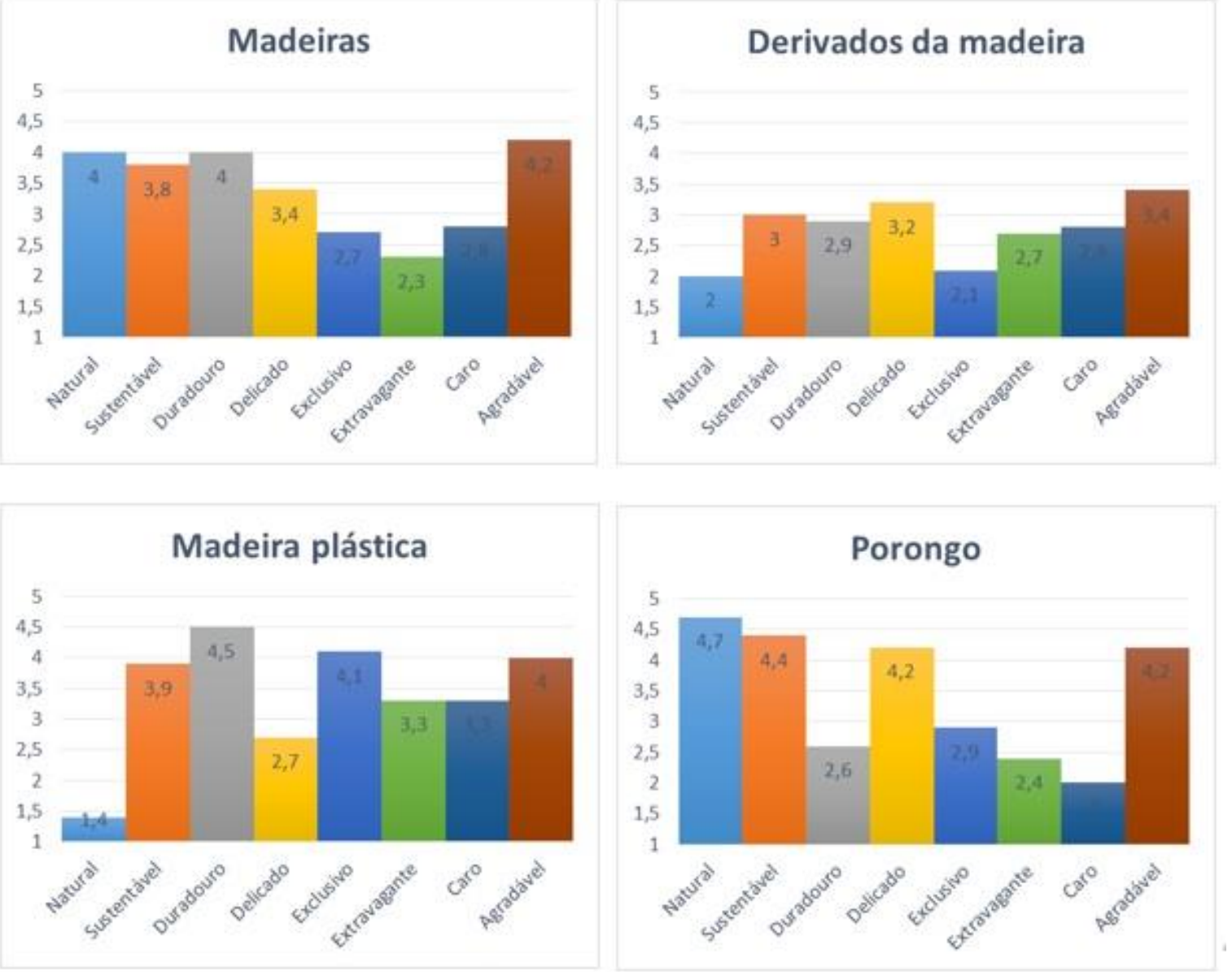

Fonte: dos autores 
A análise semântica do porongo foi muito similar à da madeira. Os atributos natural, sustentável e agradável obtiveram pontuação alta, acima de 4,0, o que reforça a relação que os participantes fazem entre estes aspectos e como eles são perceptíveis no material. $O$ atributo natural foi o que obteve a pontuação mais alta, 4,7 de um máximo de 5,0, o que reforça que o porongo é percebido como um material natural. Diferente da madeira, o atributo duradouro obteve pontuação relativamente baixa, enquanto que o atributo delicado obteve pontuação alta. A relação na escala semântica entre os atributos duradouro e delicado pode ser comparada à relação na escala sensorial entre leve e forte. É provável que o usuário tenha a compreensão de que duradouro e delicado são atributos contrários, inversamente proporcionais.

Ao analisar os resultados da percepção material do porongo a partir das duas escalas, constata-se a configuração de atributos diretamente e inversamente proporcionais. Podem ser considerados atributos diretamente proporcionais, com alta pontuação, macio, liso, leve, natural, sustentável, delicado e agradável. Assim, se o material é percebido como macio, liso e leve, ele é compreendido pelo usuário como sendo delicado e agradável, tátil e visualmente. Em contraponto, os atributos forte, duradouro e caro, com pontuação consideravelmente baixa, configuram-se como inversamente proporcionais aos primeiros. Existe a associação de que se o material é muito leve deve ser frágil e quebradiço. Ainda, por ser natural e abundante, deve ter um preço acessível para o consumidor final.

Por fim, o comparativo com a madeira é um importante parâmetro para compreender a percepção material que os usuários têm do porongo. Apesar da constante evolução na engenharia de materiais, tanto no desenvolvimento de novas matérias-primas quanto no beneficiamento das existentes, a madeira continua sendo referência em termos de materiais naturais, pelas suas propriedades físicas e estéticas e pela amplitude das possibilidades de aplicação. Assim, o fato da percepção material do porongo ser similar à madeira em aspectos determinantes demonstra o potencial do estudo do material para futuras aplicações.

\section{Considerações finais}

A crescente busca por produtos produzidos a partir de materiais naturais, menos prejudiciais ao meio ambiente durante todo o ciclo de vida, não é apenas uma tendência, mas assinala uma mudança cultural e de comportamento. Os resultados do estudo evidenciam que os participantes entendem um material natural como inerentemente sustentável e, consequentemente é percebido como mais agradável. Partindo da premissa de que o porongo é identificado como um material natural, sustentável e esteticamente agradável, e da demanda crescente por produtos ecologicamente corretos em todas as áreas, cria-se um indicativo favorável para o investimento e o esforço em desenvolver novos produtos a partir da matéria-prima.

Quanto aos resultados do estudo, com relação à escala sensorial, o atributo da leveza obteve pontuação alta tanto nas madeiras quanto no porongo, sendo o último considerado ainda mais leve que as primeiras. A percepção de que um material é quente está relacionada com a sensação de conforto do uso do mesmo. Este é um atributo característico da madeira e é muito explorado no design de móveis e ambientes. Este atributo obteve uma pontuação ligeiramente mais alta no porongo do que nas madeiras, o que é um indício positivo para o direcionamento da aplicação do material nestas áreas. 
Os resultados da escala semântica mostram que os atributos natural e sustentável estão altamente pontuados e são diretamente relacionados tanto nas madeiras quanto no porongo, sendo que o último obteve pontuação superior que a primeira. 0 porongo é percebido como igualmente agradável que a madeira, com uma pontuação de 4,2 de um máximo de 5,0. Ainda, é percebido como um material delicado, sendo a pontuação deste atributo igual à do atributo agradável.

Não obstante, cabe ressaltar que os resultados do estudo podem ter sofrido alguma interferência advinda da familiaridade dos participantes com o material, apesar de não haver identificação do nome do material nas amostras. Grande parte da população da região sul do país tem contato diário com o porongo na figura da cuia para o chimarrão. Como sugestão para estudos futuros, seria pertinente aplicar o questionário para uma amostra maior e mais diversificada, tanto em termos de gênero, idade, condição social e cultural, e oriunda de diferentes regiões do país, onde o material seja menos conhecido.

Ainda com relação a sugestões para trabalhos futuros, seria interessante comparar a percepção material do porongo com outros materiais naturais e derivados. A engenharia de materiais desenvolve constantemente novos materiais biodegradáveis, além dos muitos materiais naturais que já são explorados industrialmente, como o bambu e fibras naturais. Partindo do pressuposto de que a percepção material do porongo é positiva quanto aos atributos de natural, sustentável, leve e agradável, seria interessante estudar a percepção dos usuários com relação ao material aplicado em produtos diversos, para compreender melhor o potencial de aplicação do material em escala industrial no design de novos produtos.

\section{Referências}

ASHBY, Michael. F.; JOHNSON, Kara. Materiais e design: arte e ciência na seleção de materiais no design de produto. Rio de Janeiro: Elsevier, 2011.

BISOGNIN, D. A.; MARCHESAN, E.; AUDE, M. I. DA S. Densidade de semeadura e produtividade do porongo. Ciência Rural, Santa Maria, v. 22, n. 1, p. 15 - 19, 1992.

BISOGNIN, Dilson A. Origin and evolution of cultivated cucurbits. Revista Ciência Rural: Santa Maria, v. 32, n. 5, p. $715-723,2002$.

BISOGNIN, Dilson A.; SILVA, André. L. L. A cultura do porongo. Informe técnico, ed. Pelo Departamento de Fitotecnia do centro de Ciências Rurais (CCR) da Universidade Federal de Santa Maria (UFSM), 2004.

BURTENSHAW, Mike. The first horticultural plant propagated from seed in New Zealand: Lagenaria siceraria. New Zealand Garden Journal, 2003.

HARTZÉN, Ann-Sofie Margareta; LINDBERG, Siv Monica. The material expression of new pulp-fibre reinforced composites in relation to other material categories. In: Celebration \& Contemplation, 10th International Conference on Design \& Emotion, Amsterdam, p. 191 - 200, 2016.

KARANA, E.; NIJKAMP, N. Fiberness, reflectiveness and roughness in the characterization of natural and high quality materials. Journal of Cleaner Production, v. 68, p. 252-260, 2014.

KISTLER, Logan; et al. Transoceanic drift and the domestication of African bottle gourds in the 
Americas. PNAS, vol. 111, no 8, 2014.

LAGO, Tatiana. Caracterização e impregnação polimérica do porongo (Lagenaria siceraria) visando aplicação no design de biojóias. Dissertação (Mestrado em Design), Universidade Federal do Rio Grande do Sul, Porto Alegre, 2013.

LIMA, Marco Antônio M. Introdução aos Materiais e Processos para Designers. Rio de Janeiro: Editora Ciência Moderna Ltda., 2006.

NEJELISKI, Danieli M. O porongo (Lagenaria siceraria) como matéria-prima para a produção de recipientes: caracterização e impermeabilização. Dissertação (Mestrado em Design), Universidade Federal do Rio Grande do Sul, Porto Alegre, 2015. 